\title{
What doesn't kill them makes them stronger: An association between elongation factor $1-\alpha$ overdominance in the sea star Pisaster ochraceus and "sea star wasting disease"
}

John P. Wares, Lauren M Schiebelhut

In recent years, a massive mortality event has killed millions of sea stars, of many different species, along the Pacific coast of North America. This disease event, known as 'sea star wasting disease' (SSWD), is linked to viral infection. In one affected sea star (Pisaster ochraceus), previous work had identified that the elongation factor 1- $\alpha$ locus (EF1A) harbored an intronic insertion allele that is lethal when homozygous yet appears to be maintained at moderate frequency in populations through increased fitness for heterozygotes. The environmental conditions supporting this increased fitness are unknown, but overdominance is often associated with disease. Here, we evaluate populations of $P$. ochraceus to identify the relationship between SSWD and EF1A genotype. Our data suggest that there may be significantly decreased occurrence of SSWD in individuals that are heterozygous at this locus. These results suggest further studies are warranted to understand the functional relationship between diversity at EF1A and survival in P. ochraceus. 
1 What doesn't kill them makes them stronger: An association between

2 elongation factor $1-\alpha$ overdominance in the sea star Pisaster

3 ochraceus and "sea star wasting disease"

$4 \quad$ J. P. Wares ${ }^{1}$ and L. M. Schiebelhut ${ }^{2}$

5 1'University of Georgia, Odum School of Ecology and Department of Genetics, Athens,

6 Georgia, USA

$7 \quad$ 2University of California, School of Natural Sciences, Merced, California, USA

8 correspondence: J. P. Wares, jpwares@uga.edu

9

10

11

12

13

14

15

16

17

18

19

20

21

22

\section{3}

24

25

26

27

28

29

30

31

32

33

\section{Abstract}

In recent years, a massive mortality event has killed millions of sea stars, of many different species, along the Pacific coast of North America. This disease event, known as 'sea star wasting disease' (SSWD), is linked to viral infection. In one affected sea star (Pisaster ochraceus), previous work had identified that the elongation factor 1- $\alpha$ locus harbored an intronic insertion allele that is lethal when homozygous yet appears to be maintained at moderate frequency in populations through increased fitness for heterozygotes. The environmental conditions supporting this increased fitness are unknown, but overdominance is often associated with disease. Here, we evaluate populations of $P$. ochraceus to identify the relationship between SSWD and EF1A genotype. Our data suggest that there may be significantly decreased occurrence of SSWD in individuals that are heterozygous at this locus. These results suggest further studies are warranted to understand the functional relationship between diversity at EF1A and survival in $P$. ochraceus.

\section{Introduction}

One of the more stunning recent news stories pertaining to ocean health was the massive die-off of sea stars on both coasts of North America via a necrotic syndrome now known as sea star wasting disease (SSWD) (Hewson et al. 2014). Similar die-offs have happened in earlier decades (Becker 2006; Eckert et al. 1999), though none as extensive as in 20132014. Hewson et al. (2014) identified a candidate densovirus that is in greater abundance in diseased sea stars, and may be a causal agent; however, there is much yet to be learned. As sea stars are key predators in marine benthic ecosystems, the impacts of disease on these organisms could dramatically restructure coastal communities (Paine 1966). Thus, we address here the potential for one species to harbor heritable variation and how this variation is affected by SSWD. 
34 During disease outbreaks, biologists are keen to know whether populations will exhibit any

35 resistance to a pathogen. Thus, management studies may include surveys of genetic

36 diversity to identify the potential for evolving resistance, or genetic rescue from other

37 regions (Whiteley et al. 2015); such studies may also provide insight into the extent of

38 population structure and gene flow among regions. Following a routine analysis of genetic

39 variation in the sea star Pisaster ochraceus (Harley et al. 2006), Pankey and Wares (2009)

40 identified an insertion mutation in an intron of the elongation factor 1- $\alpha$ gene (hereafter

41 EF1A) that appeared to exhibit overdominance. In this case, the insertion is lethal when

42 homozygous (Pankey \& Wares 2009), yet the average frequency of the insertion allele was

$43 \sim 0.24$ along the Pacific coast of North America. These observations suggest that the

44 heterozygote has a significant fitness advantage in an unknown environmental setting.

45 Overdominance is often associated with resistance to disease or toxins, however, and

46 Pankey and Wares (2009), referring to what is now called SSWD, speculated that:

47

48

49

50

51

52

53

54

55

56

57

58

59

60

61

62

63

64

65

66

67

68

72

73

"widespread die-offs on the west coast of North America... could exert a substantial selective force on Pisaster. Given the prevalence of pathogen resistance in earlier studies of overdominance, we believe this to be a probable explanation for the maintenance of the described ... polymorphism."

There is concern that elevated sea temperature is a component of the SSWD outbreak (Bates et al. 2009; Eisenlord et al. 2016; Hewson et al. 2014). The relationship between expression of EF1A and thermal tolerance has been identified in other metazoans (Buckley et al. 2006; Stearns et al. 1993), and functions in part through rapid co-production of proteins associated with the heat shock response. Though Pankey and Wares (2009) were not able to detect EF1A expression differences among individuals of differing genotype, we were not able at the time to control for a number of environmental factors nor the possible action of splice variants. Here, we posit an indirect mechanistic relationship between temperature, the effect of expression of EF1A, and SSWD. With as little as is known about this disease and marine disease in general (Mydlarz et al. 2006), this is at best an educated guess. However, it is useful to know what potential $P$. ochraceus and other sea stars have for surviving this outbreak and natural patterns of genetic variation, and whether subsequent generations will be more resistant or tolerant of similar pathogens. Here we evaluate this simple polymorphism from populations of $P$. ochraceus collected prior to and following the SSWD outbreak, as well as focus on extant individuals and their disease status. We ask whether there are frequency shifts of the two genotypes at this locus that may be associated with resistance to SSWD, and evaluate efforts to explore similar genomic variation in other species.

\section{Methods}

\section{Pisaster and Disease Status}

Collections were made in 2014-2015 from locations in central California, Oregon, coastal Washington and the San Juan Islands (WA), and Nanaimo (Vancouver), and categorized by health status using the Pacific Rocky Intertidal Monitoring Network classification 
74 (http://www.eeb.ucsc.edu/pacificrockyintertidal/index.html; Table 1). Complete

75

76

77

78

79

80

81

82

83

84

85

86

87

88

89

90

91

92

93

94

95

96

97

98

99

100

101

102

103

104

105

106

107

108

109

110

111

112

113

information on collection location, individual sizes, permitting, and other metadata are in Supplemental Data S1. All permits are listed in Supplement S1. Specimens were collected under California Department of Fish and Wildlife permits \#11794 to L. Schiebelhut and \#603 to M. N Dawson, California State Parks permit to M. N Dawson, and National Parks permits PORE-2012-SCI-0038, PORE-2013-SCI-0033, and REDW-2014-SCI-0018. Collections at Nanaimo were under Dept. of Fisheries and Oceans site permit at Pacific Biological Station; tissues from the San Juan Islands were re-sampled under permits for Eisenlord et al. (2016).

\section{Temporal Comparisons}

In addition to individuals explicitly assessed for health status, we also considered the potential for genotype (and allele) frequency change following a related disease outbreak. Previous EF1A genotype/allele frequency information from specimens collected in 20032004 are available for many locations along the Pacific coast (Pankey \& Wares 2009). In central California, tissues from 4 locations (Sonoma County) were obtained in both 2012 (pre-outbreak) as well as 2014 (the tissues noted in previous section from these sites). Thus genetic frequencies from 3 time points can be assessed. Along the Oregon, Washington, and San Juan Island coastal regions, the tissues noted in previous section (from 2014-2015) can be compared to the genetic frequency information from 2003-2004 tissues.

\section{Molecular Methods}

As in Pankey and Wares (2009), primers PisEF1-F (5'-aggctgccgataccttcaa-3') and PisEF1$\mathrm{R}\left(5^{\prime}\right.$-gctagtatctgtttctgtgtgactgc-3') were used to determine individual EF1A genotypes by scoring length-polymorphic PCR products on $2 \%$ (or greater) agarose gels. About $10 \%$ of individuals were multiply genotyped so that genotype error rate (Pompanon et al. 2005) could be assessed. An example of this polymorphism is shown in Figure 1.

\section{Statistical Analyses}

Our first approach is to ask whether the frequency distribution of the two EF1A genotypes differs between diseased and healthy individuals of $P$. ochraceus. Specimens from distinct locations are grouped when sites are within $50 \mathrm{~km}$ of each other and each regional sample is evaluated separately as well as combined. Separate analyses recognize the potential for heterogeneity at related quantitative traits despite apparent phylogeographic homogeneity (Harley et al. 2006), as well as distinct environmental influences, while pooled analysis augments statistical power.

Regional and combined data are analyzed first with a Fisher's exact test. Additionally, following Gerrodette (2011), we estimate the effect size of genotype on SSWD mortality by subtracting the proportion of homozygous individuals exhibiting SSWD from the proportion of heterozygous individuals exhibiting SSWD. Here we assume that the genotype frequencies are binomially distributed (with associated sampling error) and estimate the difference in proportion of disease incidence (also here assumed binomial) 
114 between homozygotes and heterozygotes. Again, these probability distributions are 115 estimated for each individual regional/temporal sample. These same statistical methods 116 were applied to evaluate genotype frequency changes between population samples from 117 before and after the 2013-14 SSWD outbreak, as described above.

118 To evaluate the probability that heterozygous individuals have a higher probability of 119 avoiding or surviving SSWD, logistic regression of the complete dataset is performed with models that incorporate individual size (when available, measured from center of disk to tip of an arm), genotype at EF1A, and region of collection. Models of single factors were evaluated, and for factors that exhibit significant variation additive and interaction models were also evaluated and compared using AIC values. The model with greatest AIC weight is considered to explain most of the variation in the system. All statistical analyses were performed using R (R_Development_Core_Team 2009).

127 The genotype error rate was 0 for 21 individuals (out of 228 in this study) that were 128 repeatedly genotyped. Two individuals initially presented a very faint second band on gel, 129 but subsequent repeat amplification of one of these confirmed it as homozygous (note: this 130 polymorphism has been assessed as an overdominant Mendelian locus (Pankey \& Wares 2009), so we do not think this represents variation in paralog amplification success).

Data from each location (Table 1) were analyzed with a basic Fisher's exact test. Each regional sample was in the predicted direction with a higher likelihood of SSWD among homozygotes than heterozygotes; no single location or regional grouping presented contrasts that were statistically significant (results not shown). Certainly a component of individual regional/temporal samples is that with modest sample sizes, statistical power is low. Combining all samples leads to a statistically significant result ( $\mathrm{p}$-value 0.0035).

When considering all available information related to disease risk in our samples, models representing single factors (size, sample location, and genotype) and combinations of factors were compared using AIC against a null (no factor) model. Size (radius) was not significant and was dropped from subsequent analyses, while location $(p<0.001)$ and genotype were both significant factors $(\mathrm{p}=0.00615)$. Given the remaining main effects and interactions, we found the AIC weight was strongest for models with both factors included (AIC weight 0.226 ) and with both factors included with interaction between (AIC weight $0.756)$. When both factors are included, both are still significant $(\mathrm{p}<0.01)$; when the model includes interactions, genotype is important but the additional parameterization reduces power $(p=0.0526)$, location is not significant, and the interaction term is not significant $(\mathrm{p}=0.0694)$. A small sample of individuals $(\mathrm{n}=14)$ had no size measurement available (Supplement S1) but exhibited no significant effect (effect=0.05, p=1) of genotype.

Despite the hypothesis of increased fitness for EF1A heterozygotes under these conditions, the frequency of the insertion (ins) allele in central California only appears to decrease through time, from approximately $0.27(n=33)$ in 2003-2004 (Pankey \& Wares 2009) to $0.24(\mathrm{n}=40)$ in 2012 and $0.25(\mathrm{n}=40)$ in 2014-2015. However, with sampling error these 
154 frequencies are statistically unchanged and a larger sample comparison may be necessary 155 to explore this component of our evaluation. In the San Juan Islands, the frequency of the 156 ins allele is also statistically unchanged from 0.27 in $2003(n=62)$ to 0.253 in $2014(n=75)$, 157 again not supporting a hypothesis of selection increasing or maintaining the ins frequency 158 (but also not a statistically significant difference in frequency). The same can be said for 159 contrasts along the Oregon and Washington coasts, where the overall frequency is 160 effectively unchanged.

\section{Discussion}

162 Our data show that our hypothesis for a relationship between disease status (SSWD) and 163 an apparent overdominant polymorphism in P. ochraceus is strongly supported-results 164 from each sample are in the predicted direction, and overall there is clear evidence that 165 sick individuals are more likely to be EF1A homozygotes than heterozygotes. The net effect 166 size of 0.19 (Table 1 ) is very similar to the fitness differential between genotypes $(\sim 0.2)$ 167 proposed by Pankey and Wares (2009) given simulations of overdominance. The variation 168 in effect seen among regional samples-and the apparent statistical interaction between sample location and genotypic effect on disease status-is likely influenced both by modest sample sizes as well as distinct exposure histories. It is likely that each of our regional samples has been exposed to distinct temperature profile histories (Bates et al. 2009; Eisenlord et al. 2016), and it is possible that despite apparent population genetic homogeneity (Harley et al. 2006) that undetected evolutionary changes have led to distinct

174

175

176 reaction norms among regional samples. Additionally, the timing of arrival and effect of the candidate densovirus that may cause SSWD may lead to distinct evolutionary dynamics across regions.

177

178

179

In previous work (Bates et al. 2009; Hewson et al. 2014) it has been evaluated whether size was a predictive factor in disease status; both research groups found no clear statistical association (though it appeared there is a negative relationship between densovirus abundance and size in Pycnopodia helianthoides; Hewson et al. 2014). A more recent analysis (Eisenlord et al. 2016) does show a significant relationship between elevated temperature exposure, as well as size, and SSWD in P. ochraceus. Our smaller sample presents no clear association between sea star radius and disease status nor an interaction with EF1A genotype.

\section{Evolutionary Response}

186

187

188

189

190

191

192

193
Despite the apparent and predicted effect in our samples, we do not see the hypothesized evolutionary response-the frequency of the ins mutation has apparently not increased in recent years. If EF1A in P. ochraceus truly evolves via overdominance, where the heterozygote is significantly more fit under certain environmental conditions, then we would expect this allele to increase in frequency when exposed to a relevant mass mortality event. However, it is also not entirely clear what proportion of individuals have died in recent years as a result of SSWD (though estimates from intertidal surveys are high enough that some frequency response is warranted; see Eisenlord et al. 2016). Thus, 
194 detection of this change could be masked in part by simple stochastic changes (e.g. genetic 195 drift) in local populations of $P$. ochraceus, and the sample size available from all three time 196 points limits our statistical power to address this hypothesis. Of course, there are other 197 forms of mortality in sea stars like P. ochraceus (Jurgens et al. 2015), and so it is still likely 198 that we are seeing an indirect interaction between recent die-offs and individual-level 199 responses that appear to be genotype dependent. Preliminary evidence (L.S., unpublished 200 observations) suggest higher frequency of the ins allele at some California sites, and some 201 of our sampled locations had higher frequencies of SSWD after tissues were harvested. 202 Thus further study, tracking frequency of the EF1A ins polymorphism through time with 203 detailed information on disease and other environmental factors at local sites, is 204 warranted.

\section{Disease in Sea Stars}

206

207

208

209

210

211

212

213

214

215

216

217

218

219

220

221

222

223

224

225

226

227

228

229

230

231

232

233

234

With limited understanding of immune response in most echinoderms (Mydlarz et al. 2006), the problem of SSWD is difficult enough to explore in P. ochraceus, let alone the many other species affected in the recent outbreak. In another species of sea star $(P$. helianthoides), Fuess et al. (2015) have identified some of the genomic components that are upregulated in response to viral exposure; however, we know of no similar (apparent) overdominant system in these other asteroids, and as yet still know very little about how this polymorphism in P. ochraceus influences EF1A expression, alternate splicing events, or what genes may be linked to this region and thus affected. Although some studies have indicated a relationship between elongation factor expression and thermal tolerance in plants (e.g., Bukovnic et al. 2009) there is still little information in metazoans (Buckley et al. 2006; Stearns \& Kaiser 1993; Stearns et al. 1993) though these studies suggest that differential expression is associated with increased lifespan and increased tolerance to heat stress. As this gene is considered "housekeeping" in many studies it is actually used to normalize the differential expression results of other genes (Fangue et al. 2006); our study suggests further attention to differential expression of this locus is warranted.

Our interest in exploring this particular case has little to do with solving the problem of disease, and more about the question of what demographics will be like for $P$. ochraceus in an increasingly warmer-and disease-affected-environment (Eisenlord et al. 2016; Harvell et al. 2002). If disease like SSWD interacts with the EF1A polymorphism as noted here, and the frequency of the deleterious ins allele increases, this could also indicate increased reproductive loss through homozygous lethality, which could decrease the potential for populations to rebound from crashes.

\section{Parallels with Malaria}

Aidoo et al. (2002) note that "sickle cell trait" in humans (carrying a single $S$ allele of hemoglobin) provides $\sim 60 \%$ protection against overall mortality, mostly in the first 16 months of life; being a carrier is not a guarantee against infection. Other studies have focused on specific malarial parasites, and note that children heterozygous for the $S$ hemoglobin allele have approximately one-tenth the mortality risk from Plasmodium falciparum as those homozygous for normal alleles (Cholera et al. 2008). In the absence of 
235 cohort data, it is difficult to estimate the level of disease or mortality protection that any

236 single polymorphism can provide (Aidoo et al. 2002). Until such recent studies, the claim of

237 overdominant selection on hemoglobin genotypes, based on the relationship between the

238 frequency of the $S$ allele and the prevalence of malaria (Allison 1954), was only correlative.

239 This relationship is still a clear case of overdominance (Gemmell \& Slate 2006), but is

240 illustrative that increased heterozygote fitness does not require absolute protection against

241 the associated risk factor-e.g., that all individuals of P. ochraceus with SSWD would be

242 homozygous for the wild-type EF1A allele identified in Pankey and Wares (2009), nor that

243 healthy individuals would all be heterozygotes - as there are many components to disease

244 avoidance, tolerance, or resistance.

\section{Conclusions}

246 At this time, with limited sampling (and recognizing that our samples themselves may not

247 be random from populations of $P$. ochraceus), our results suggest an intriguing (but

248 probably indirect) relationship between SSWD susceptibility and the EF1A polymorphism

249 described. The direction of effect is consistent in all subsamples, and the magnitude of

250 effect overall is comparable to predictions based on simulations of overdominance in this

251 system given the observed frequency of the ins allele and lethality of ins homozygotes

252 (Pankey \& Wares 2009). Nevertheless, we do not see an increase in the frequency of the ins

253 allele over time in our samples and so we remain curious about the dynamics of

254 overdominance in this system.

255 It is possible that regulation and expression of EF1A is influenced by this polymorphism in

256 a way that alters an individuals' tolerance or capacity for heat stress, and in a warming

257

258

259

260

261

262

263

264

265

266

267

268

269

270 climate and ocean it is known that disease and mortality are higher in large part because of physiological stress modifying an organism's response to pathogens (Eisenlord et al. 2016; Harvell et al. 2002). Further work is needed not only to examine the association shown here, but also to identify (i) whether size or maturity is truly important in this relationship, (ii) whether individuals of different genotype do have distinct constitutive or regulated patterns of expression of EF1A or related/linked genes, and (iii) whether there are genotype-driven differences in mortality of individuals under thermal stress (which can affect feeding rates as well as physiological factors in P. ochraceus; (Sanford 2002)).

In the meantime, we emphasize that this system is so easy to explore as a low-budget research project or teaching tool that there are opportunities to work as a community to greatly expand our understanding of the maintenance of the overdominant EF1A diversity in P. ochraceus, perhaps for other pertinent variables of interest. We would encourage any future studies to ensure that sufficient metadata are associated with any such comparison so that this relationship can continue to be explored.

\section{Acknowledgments}

272 The authors would like to thank Collin Closek (Wares Lab alumnus), Mo Turner, and

273 Morgan Eisenlord for coordinating tissue samples from FHL; Mike Hart, Vanessa Guerra,

274 and David Breault for specimen collection and handling of Nanaimo tissues in 2015; and 
275 Peter Raimondi and PISCO for other coastal samples collected in 2014-2015. We thank

276 Sarah Abboud, Charlsie Berg, Anny Calderon, Lorely Chavez, Brendan Cornwell, Michael N.

277 Dawson, Madlen Friedrich, Brian Gaylord, Alehandra Guzman, Brittany Jellison, Laura

278 Jurgens, Shawn Knapp, Kelly McClintock, Holly Mondo, Mira Parekh, Emily Ramirez,

279 Mariana Rocha de Souza, Adam Rosso, Stephen Sanchez, Holly Swift, Sabah Ul Hussan, and

280 Jesse Wilson for assisting with California collections in 2012-2014. Tim Makinde generated

281 much of the genotypic data at UGA. Members of the Wares Lab at UGA and colleagues at the

282 Odum School of Ecology, particularly John Drake, assisted greatly with discussion of the

283 idea and early drafts of the manuscript. The manuscript also benefited from constructive

284 comments by Morgan Eisenlord, Associate Editor Keith Crandall, and three anonymous

285 reviewers. We also thank Sarah Graven for organizing the SSWD-themed session at the

286 Western Society of Naturalists meeting in 2015 from which we gained perspective and

287 useful comments on this project.

288

289

290

291

292

293

294

295

296

297

298

299

300

301

302

303

304

305

306

307

308

309

310

311

312

\section{References}

Aidoo M, Terlouw DJ, Kolczak M, McElroy PD, ter Kuile FO, Kariuki S, Nahlen BL, Lal AA, Udhayakumar V (2002) Protective effects of the sickle cell gene against malaria morbidity and mortality. Lancet 359, 1311-1312.

Allison AC (1954) The Distribution of the Sickle-Cell Trait in East Africa and Elsewhere, and Its Apparent Relationship to the Incidence of Subtertian Malaria. Transactions of the Royal Society of Tropical Medicine and Hygiene 48, 312-318.

Bates AE, Hilton BJ, Harley CD (2009) Effects of temperature, season and locality on wasting disease in the keystone predatory sea star Pisaster ochraceus. Diseases of Aquatic Organisms 86, 245-251.

Becker BJ (2006) Status and Trends of Ecological Health and Human Use of the Cabrillo National Monument Rocky Intertidal Zone (1990-2005) (ed. Service NP). U.S. Dept. of the Interior

Buckley BA, Gracey AY, Somero GN (2006) The cellular response to heat stress in the goby Gillichthys mirabilis: a cDNA microarray and protein-level analysis. Journal of Experimental Biology 209, 2660-2677.

Bukovnic U, Fu J, Bennett M, Prasad PVV, Ristic Z (2009) Heat tolerance and expression of protein synthesis elongation factors, EF-Tu and EF- $1 \alpha$, in spring wheat. Functional Plant Biology 36, 234-241.

Cholera R, Brittain NJ, Gillriet MR, Lopera-Mesa TM, Diakite SAS, Arie T, Krause MA, Guindo A, Tubman A, Fujioka H, Diallo DA, Doumbo OK, Ho M, Wellems TE, Fairhurst RM (2008) Impaired cytoadherence of Plasmodium falciparum-infected erythrocytes containing sickle hemoglobin. Proceedings of The National Academy Of Sciences of The United States Of America 105, 991-996. 
313 Eckert GL, Engle JM, Kushner DJ (1999) Sea star disease and population declines at the Channel Islands. 5, 390-393.

315

316

317

318

319

320

321

322

323

324

325

326

327

328

329

330

331

332

333

334

335

336

337

338

339

340

341

342

343

344

345

346

347

348

349

350

Eisenlord ME, Groner ML, Yoshioka RM, Elliott J, Maynard J, Fradkin S, Turner M, Pyne K, Rivlin N, van Hooidonk R, Harvell CD (2016) Ochre star mortality during the 2014 wasting disease epizootic: role of population size structure and temperature. Philosophical Transactions of the Royal Society B 371, 20150212.

Fangue NA, Hofmeister M, Schulte PM (2006) Intraspecific variation in thermal tolerance and heat shock protein gene expression in common killifish, Fundulus heteroclitus. Journal of Experimental Biology 209, 2859-2872.

Fuess LE, Eisenlord ME, Closek CJ, Tracy AM, Mauntz R, Gignoux-Wolfsohn S, Moritsch MM, Yoshioka R, Burge CA, Harvell CD, Friedman CS, Hewson I, Hershberger PK, Roberts SB (2015) Up in Arms: Immune and Nervous System Response to Sea Star Wasting Disease. PLoS ONE 10, e0133053.

Gemmell NJ, Slate J (2006) Heterozygote advantage for fecundity. PLoS ONE 1, e125.

Gerrodette T (2011) Inference without significance: measuring support for hypotheses rather than rejecting them. Marine Ecology-an Evolutionary Perspective 32, 404-418.

Harley CDG, Pankey MS, Wares JP, Grosberg RK, Wonham MJ (2006) Color polymorphism and genetic stucture in the sea star Pisaster ochraceus. Biological Bulletin 211, 248262.

Harvell CD, Mitchell CE, Ward JR, Altizer S, Dobson AP, Ostfeld RS, Samuel MD (2002) Ecology - Climate warming and disease risks for terrestrial and marine biota. Science 296, 2158-2162.

Hewson I, Button JB, Gudenkauf BM, Miner B, Newton AL, Gaydos JK, Wynne J, Groves CL, Hendler G, Murray M, Fradkin S, Breitbart M, Fahsbender E, Lafferty KD, Kilpatrick AM, Miner CM, Raimondi P, Lahner L, Friedman CS, Daniels S, Haulena M, Marliave J, Burge CA, Eisenlord ME, Harvell CD (2014) Densovirus associated with sea-star wasting disease and mass mortality. Proceedings Of The National Academy of Sciences Of The United States Of America 111, 17278-17283.

Jurgens LJ, Rogers-Bennett L, Raimondi PT, Schiebelhut LM, Dawson MN, Grosberg RK (2015) Patterns of Mass Mortality among Rocky Shore Invertebrates across $100 \mathrm{~km}$ of Northeastern Pacific Coastline (vol 10, e0126280, 2015). PLoS ONE 10.

Mydlarz L, Jones L, Harvell C (2006) Innate immunity, environmental drivers, and disease ecology of marine and freshwater invertebrates. Annual Review of Ecology, Evolution, and Systematics 37, 251-288.

Paine RT (1966) Food web complexity and species diversity. American Naturalist 100, 6575.

Pankey MS, Wares JP (2009) Overdominant maintenance of diversity in seastar Pisaster ochraceus. Journal of Evolutionary Biology 22, 80-87. 
351 Pompanon F, Bonin A, Bellemain E, Taberlet P (2005) Genotyping errors: causes, consequences and solutions. Nature Reviews Genetics 6, 847-859.

Whiteley AR, Fitzpatrick SW, Funk WC, Tallmon DA (2015) Genetic rescue to the rescue.

Trends in Ecology \& Evolution 30, 42-49.

R Development Core Team (2009) R: A language and environment for statistical computing. R Foundation for Statistical Computing, Vienna, Austria.

Sanford E (2002) The feeding, growth, and energetics of two rocky intertidal predators (Pisaster ochraceus and Nucella canaliculata) under water temperatures simulating episodic upwelling. Journal of Experimental Marine Biology and Ecology 273, 199218.

Stearns SC, Kaiser M (1993) The effects of enhanced expression of elongation factor EF-1 alpha on lifespan in Drosophila melanogaster. IV. A summary of three experiments. Genetica 91, 167-182.

Stearns SC, Kaiser M, Hillesheim E (1993) Effects on Fitness Components of Enhanced Expression of Elongation-Factor Ef-1-Alpha in Drosophila-Melanogaster .1. The Contrasting Approaches of Molecular and Population Biologists. American Naturalist 142, 961-993.

369 


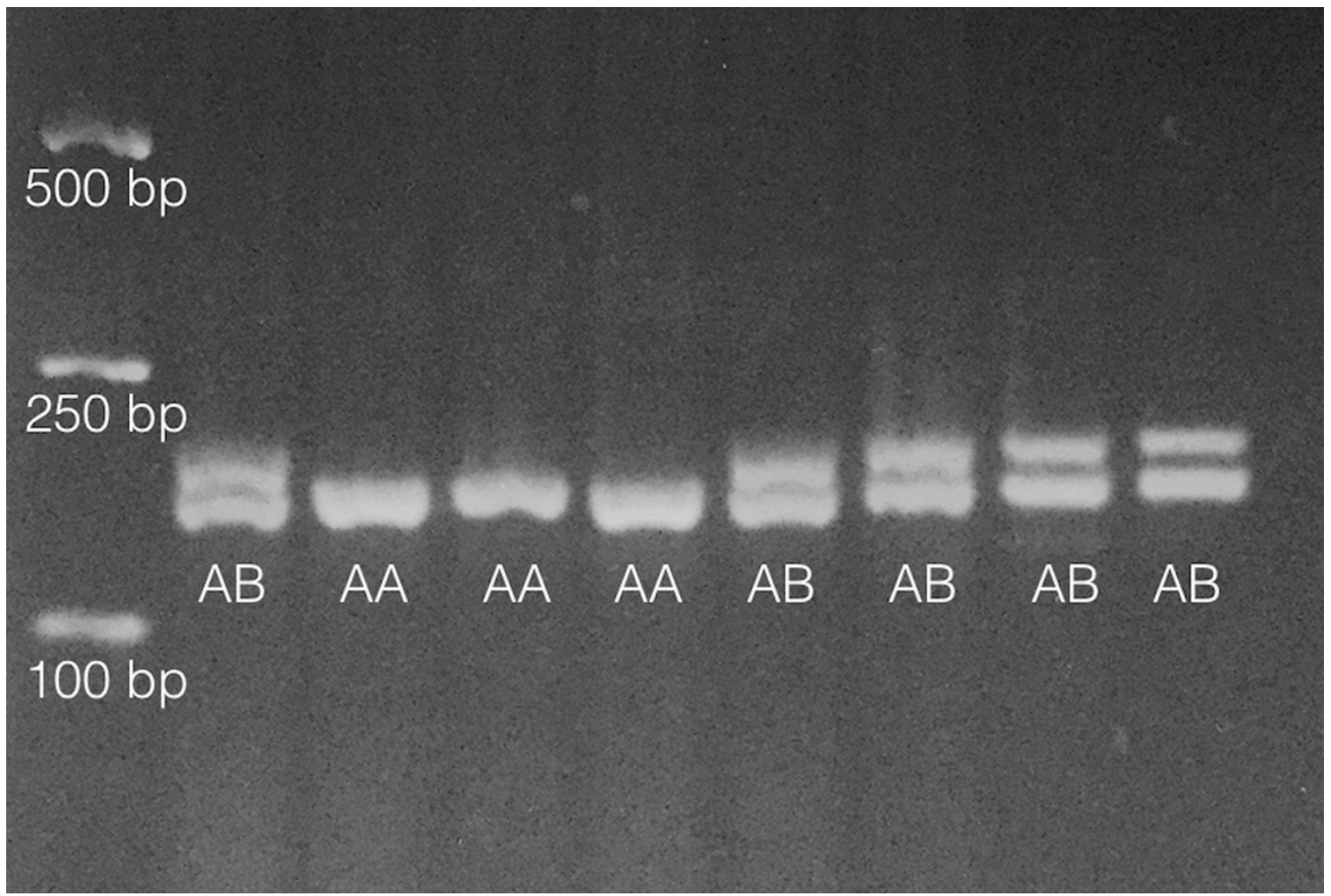

372 Figure 1. Results from analysis of 8 individuals on $2 \%$ agarose gel following PCR

373 amplification as noted in Methods. Fragments only vary by 6bp in length so gel must be run 374 for $\sim 60$ minutes under typical conditions ( $90-100 \mathrm{~V})$. Heterozygotes are denoted 'AB' and 375 homozygotes denoted 'AA' on gel image. Size standards are on left side of gel. 


\begin{tabular}{|c|c|c|c|c|c|c|c|}
\hline Site/Region & Latitude & Longitude & $\begin{array}{l}\text { SSWD } \\
\text { symptomatic } \\
+/+\end{array}$ & $\begin{array}{l}\text { SSWD } \\
\text { symptomatic } \\
+/ \text { ins }\end{array}$ & $\begin{array}{l}\text { SSWD } \\
\text { asymptomatic } \\
+/+\end{array}$ & $\begin{array}{l}\text { SSWD } \\
\text { asymptomatic } \\
+/ \text { ins }\end{array}$ & $\begin{array}{l}\text { Effect } \\
\text { Size }\end{array}$ \\
\hline Nanaimo, Vancouver, BC & 49.2 & 124 & 8 & 4 & 7 & 5 & 0.089 \\
\hline Olympic Peninsula, WA & 48.5 & 125 & 5 & 0 & 11 & 4 & 0.31 \\
\hline San Juan Island, WA & 48.5 & 123 & 25 & 17 & 15 & 18 & 0.14 \\
\hline Cape Meares, OR & 45.5 & 124 & 1 & 0 & 4 & 5 & 0.2 \\
\hline Seal Rock, OR & 44.5 & 124.1 & 1 & 2 & 1 & 6 & 0.25 \\
\hline Coquille Point, OR & 43.1 & 124.4 & 7 & 0 & 1 & 2 & 0.88 \\
\hline Damnation Creek, CA & 41.7 & 124.1 & 1 & 1 & 4 & 4 & 0 \\
\hline Sonoma County, CA & 38.7 & 123.4 & 8 & 1 & 22 & 18 & 0.21 \\
\hline San Francisco Bay, CA & 38.0 & 122.8 & 0 & 0 & 7 & 13 & 0 \\
\hline Overall & & & 56 & 25 & 72 & 75 & 0.19 \\
\hline
\end{tabular}

Table 1. Sample sizes from each regional collection of individuals (see Supplemental Table 1 for additional sampling information); samples are listed by health status as well as genotype (+/+ wild type, +/ins for the heterozygote genotype). Effect size refers to the difference in proportion of EF1A homozygotes that exhibit SSWD and the proportion of heterozygotes with SSWD; positive numbers suggest a higher proportion of homozygotes with SSWD. 